\title{
Spatiotemporal image correlation analysis of blood flow in branched vessel networks of zebrafish embryos
}

Nicolo G. Ceffa

Ilaria Cesana

Maddalena Collini

Laura D'Alfonso

Silvia Carra

Franco Cotelli

Laura Sironi

Giuseppe Chirico 


\title{
Spatiotemporal image correlation analysis of blood flow in branched vessel networks of zebrafish embryos
}

\author{
Nicolo G. Ceffa, ${ }^{a}$ Ilaria Cesana, ${ }^{a}$ Maddalena Collini, ${ }^{a, b, c}$ Laura D'Alfonso, ${ }^{a}$ Silvia Carra, ${ }^{d}$ Franco Cotelli, ${ }^{d}$ \\ Laura Sironi, ${ }^{a, \star}$ and Giuseppe Chirico ${ }^{a, b, c, *}$ \\ aUniversità di Milano-Bicocca, Dipartimento di Fisica, Milano, Italy \\ 'Institute of Applied Sciences and Intelligent Systems, CNR-ISASI, Pozzuoli, Italy \\ 'Università di Milano-Bicocca, Nanomedicine Center, Monza, Italy

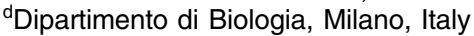

\begin{abstract}
Ramification of blood circulation is relevant in a number of physiological and pathological conditions. The oxygen exchange occurs largely in the capillary bed, and the cancer progression is closely linked to the angiogenesis around the tumor mass. Optical microscopy has made impressive improvements in in vivo imaging and dynamic studies based on correlation analysis of time stacks of images. Here, we develop and test advanced methods that allow mapping the flow fields in branched vessel networks at the resolution of 10 to $20 \mu \mathrm{m}$. The methods, based on the application of spatiotemporal image correlation spectroscopy and its extension to cross-correlation analysis, are applied here to the case of early stage embryos of zebrafish. @ 2017 Society of Photo-Optical Instrumentation Engineers (SPIE) [DOI: 10.1117/1.JBO.22.10.106008]
\end{abstract}

Keywords: image correlation; hemodynamics; single plane illumination microscopy.

Paper 170462R received Jul. 17, 2017; accepted for publication Sep. 26, 2017; published online Oct. 13, 2017.

\section{Introduction}

Flow regulation of physiological activity is a wide field of life sciences. ${ }^{1}$ Both in human beings and artificial environments, three-dimensional (3-D) structures such as cellular aggregates leading to organoids or bacterial colonies are often immersed in an aqueous fluid in motion and subjected to hydrodynamic forces. ${ }^{2}$ The progression of a wide number of diseases is affected directly by blood circulation. An important example is cancer ${ }^{3}$ and its metastases. Some diseases produce an impaired blood supply to organs. ${ }^{4,5}$ These different fields of research share a common feature. The fluid circulation presents a pulsatile, occasionally irregular, flow with ramifications in evolution, and it requires high spatial and time resolution to be studied.

The focus of the field of angiogenesis research has, in recent years, shifted toward the analysis of network formation mechanisms, also connected to tissue engineering, ${ }^{6}$ as well as the study of vessel maturation and remodeling processes. ${ }^{7}$ This has led to the discovery of vascular guidance and networking molecules as well as the identification of vessel maturation-regulating molecules, such as the angiopoietins and the platelet-derived growth factors. ${ }^{8}$ In this field, medical research often exploits small animal models such as zebrafish embryos ${ }^{9}$ and juvenile transparent fishes ${ }^{10,11}$ for pilot in vivo studies. In addition, the advances of microfluidics are greatly influencing several fields of microbiology, providing new tools to investigate processes developing under flow, such as bacterial biofilm formation. ${ }^{12}$

Our aim is, therefore, to devise methods to map quantitatively the flow field at the branching point along a vessel network. We describe and test an algorithm able to quantify in a user-independent way the dominant flow in a branched network

*Address all correspondence to: Laura Sironi, E-mail: laura.sironi@unimib.it; Giuseppe Chirico, E-mail: Giuseppe.chirico@unimib.it of sprouting vessels. To show real-field applications, we apply these algorithms on the zebrafish embryo model by following the motion of red blood cells in the embryo's blood (contrary to hemolymph, which is the fluid circulating in arthropods). Our method is based on the use of spatiotemporal image correlation spectroscopy (STICS) analysis coupled to single plane illumination microscopy (SPIM). ${ }^{13}$ This strategy allows us to reach flow field mapping with the spatial resolution of a few micrometers and to single out different periodic components, as typically found in hemodynamics. ${ }^{9,14}$ A powerful alternative technique, Doppler optical coherence tomography, has been applied $^{15,16}$ to map the flow field in branched microfluidic devices in 3-D (10- $\times 10-\times 10-\mu \mathrm{m}^{3}$ resolution). These works, however, were focused on the study of turbulence in large channels ( $\mathrm{mm}$ in size) and at larger flow speeds (few $\mathrm{mm} / \mathrm{s}$ ) than typically found in hemodynamics. Cross-correlation analysis was already applied on a dual or multispot level for the detailed analysis of the flow in straight microfluidic capillaries and in straight vessels in vivo. ${ }^{9}$ STICS analysis was applied to wide field images of blood flow in vessels as a validation for intrasurgical applications. ${ }^{17}$ We extend here the original STICS algorithm ${ }^{18}$ to the cross correlation over different fields of view chosen along the flow direction to increase the dynamic range for the measurement of the flow speed and use a multicomponent analysis of the correlation maps that allows us to exploit the amplitude of the correlation map to evaluate the flow fractionation at the branching points. The present analysis is limited here to twodimensional (2-D) flows that can be analyzed simply by following the displacement of the cross-correlation map with the lag time. However, our STICS-based approach can be extended to a 3-D reconstruction of the flow field by following the crosscorrelation amplitude decay with the lag time.

1083-3668/2017/\$25.00 @ 2017 SPIE 


\section{Experimental Section}

\subsection{Single Plane Illumination Microscopy Setup}

The SPIM setup ${ }^{18,14}$ employs an argon-krypton laser (Melles Griot), expanded 1:4 and focused into the back aperture of an illumination objective (Olympus, UMPLFLN 10XW, 10×, $\mathrm{NA}=0.3$, working distance $=3.5 \mathrm{~mm}$ ). The fluorescence emission is selected by a bandpass filter and is detected by an EMCCD detector (Cascade II, Photometrics) through an Olympus MPLFLN objective $(20 \times, \mathrm{NA}=0.5$, working distance $=3.5 \mathrm{~mm}$; see Fig. 1 for details). The maximum acquisition rate was $4 \mathrm{~ms} /$ frame on reduced regions of $40 \times 250 \mu \mathrm{m}^{2}$.

\subsection{Zebrafish Embryos}

The measurements were done on embryos of zebrafish (3 days postfertilization, d.p.f.). We used the transgenic line mitfa $^{\mathrm{w} 2 / \mathrm{w} 2}$; roy $^{\mathrm{a} 9 / \mathrm{a} 9} ; \mathrm{Tg}(\mathrm{kdrl}: \mathrm{EGFP})^{\mathrm{S} 843} ; \mathrm{Tg}-(\text { gata1 }: \mathrm{dsRed})^{\mathrm{sd} 2}$, carrying green-labeled epithelium (EGFP) and red-labeled (dsRed) red blood cells. ${ }^{19}$ The zebrafish embryos were anesthetized ${ }^{20}$ with tricaine [40 mg/L tricaine(ethyl 3-aminobenzene methansulfonate, Sigma-Aldrich Corporation, St. Louis, Missouri)], positioned in a 2-mm diameter fluorinated ethylene propylene tube (FT2X3, Adtech Polymer Engineering, Frampton Mansell, UK), and then immersed in a water cell. ${ }^{14,21}$

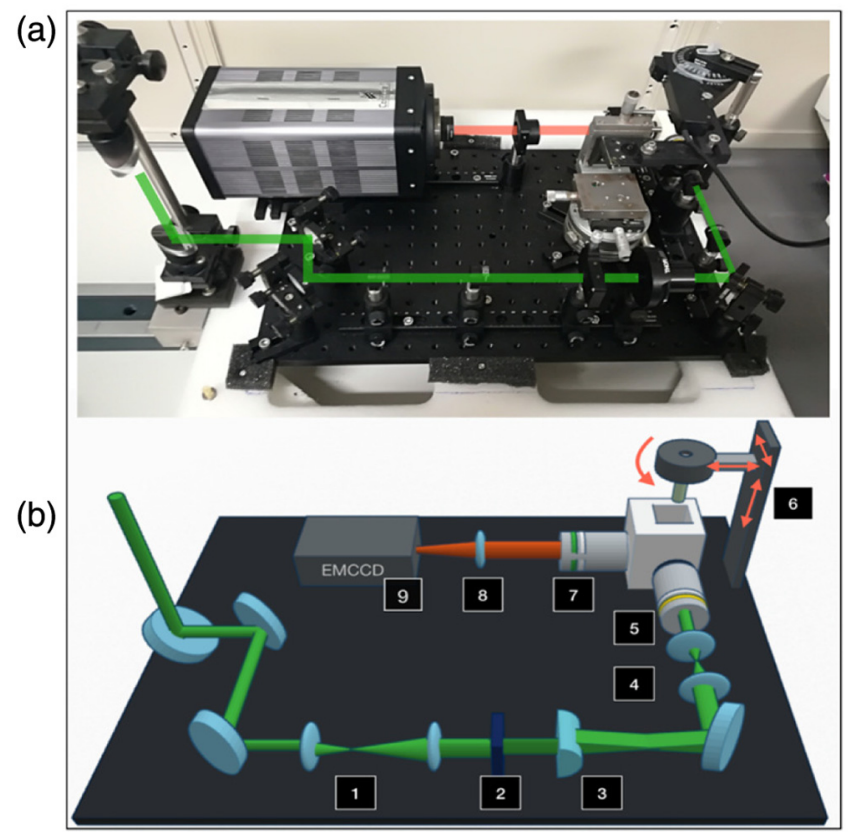

Fig. 1 (a) Photo and (b) sketch of the SPIM setup. The source is an argon laser (514-nm emission line). A beam expander (1, focal lengths 25 and $50 \mathrm{~mm}$ ) expands the incoming laser beam. A variable rectangular slit (2) creates a thin vertical aperture in the optical path. The cylindrical lens $(3)$ is positioned at the focal length $(50 \mathrm{~mm})$ from the slit. The beam reducer (4, focal lengths 50 and $25 \mathrm{~mm}$ ) reduces the incoming laser beam to fill the objective back aperture. The excitation objective (5) is an Olympus, water dipping, 10x, 0.3 NA. (6) The positioning system (for sample rotation and 3-D translation) and the sample holder. The detection objective (7) is an Olympus, water dipping, 20x, 0.5 NA. The tube lens (8) has focal length $150 \mathrm{~mm}$. The detector (9) is an EMCCD detector (Cascade II-photometrics).

\subsection{Spatiotemporal Image Correlation Spectroscopy Images Analysis}

The STICS images were computed on a time stack of $M$ images $S=\left\{I_{j}\right\}_{j=0, \ldots, M}$ taken at $M$ times spaced by $\delta t$ ranging typically from 13 to $19 \mathrm{~ms}$ (corresponding to 74 to 52 frames/s of the EMCCD, the fastest rates compatible with the chosen field of view). We take the SPIM plane as the $x-y$ plane. We assume that the flow velocity lies in this plane and is $\vec{v}=\left(v_{x}, v_{y}\right)$. Each image has a size of $X \times Y$ pixels. The image autocorrelation function was computed on the fluctuation matrices obtained by subtracting pixel by pixel the average signal $\left\langle I_{k}(x, y)\right\rangle_{k}=\sum_{k=1}^{M} I_{k}(x, y) / M$ to the content of each image of the stack, $\delta I_{j}(x, y)=I_{j}(x, y)-\left\langle I_{k}(x, y)\right\rangle_{k}$, by the FFT algorithm as follows:

$$
G(\xi, \eta ; k \delta t)=\sum_{j=0}^{M-k} \operatorname{FFT}^{-1}\left[\operatorname{FFT}\left(\delta I_{j}\right) \operatorname{FFT}^{*}\left(\delta I_{j+k}\right)\right] /(M-k)
$$

Each image of the time stack was padded with zeros up to double its size before performing the FFT.

On each correlation image, corresponding to the lag time $\tau$, we automatically selected the position $\left(\xi_{\max }, \eta_{\max }\right)$ of the maximum by fitting the image to a 2-D Gaussian profile. The amplitude of the STICS function was derived from the amplitude of the Gaussian fit function, subtracted for the possible background.

Even though the morphological images are available, we do not want to presume any a priori knowledge of the flow direction. We have then simply computed the STICS image on rectangular regions of interest (ROIs) aligned with the axes of the image as it is acquired. To evaluate the components of the flow velocity in the SPIM plane, we follow the position of the STICS image with the lag time over at least 5 to 10 lag time points, up to a lag time $\tau_{\text {fit }}$. We have checked that the condition to recover the STICS amplitude is that the ROI size is at least $S_{\mathrm{ROI}} \cong 2 v_{x y} \tau_{\mathrm{fit}}$. A flow of the order $400 \mu \mathrm{m} / \mathrm{s}$ and $\tau_{\text {fit }} \cong 40 \mathrm{~ms}$ corresponds to a size of $16 \mu \mathrm{m}$, or at least 20 pixels. This is our spatial limitation in the reconstruction of the field flow.

\section{Results and Discussion}

\subsection{Spatiotemporal Image Correlation Spectroscopy Allows to Follow the Flow Along Curved Vessels}

We want to devise methods to map the blood flow in tiny branched capillaries. The flow should be characterized in terms of direction and amplitude of the velocity, and we will need to evaluate the flow branching ratio at the ramifications along the network. The rationale of the methods that we are describing is to autocorrelate and cross correlate in space and time stacks of images acquired through a wide field SPIM. In general, we could also map the velocity field by tracking, and then averaging, many trajectories of individual red blood cells. This is not always feasible, for example, when the vessel morphology cannot already be singled out from the images (initial angiogenesis) or the heart pulsation affects the flow, such as in arteries. STICS offers the advantage of directly providing an average flow map in which the heart pulsation effect is averaged out. With STICS, we obtain from a time stack of images a series of autocorrelation maps 
[Eq. (1)], $G(\xi, \eta ; \tau)$, function of the lag time $\tau$. For a uniform drift, the map assumes the shape of a 2-D quasi-Gaussian ${ }^{13,14}$ function whose maximum occurs at the position $\vec{r}_{\max }=\left(\xi_{\max }, \eta_{\max }\right)$, measured with respect to the origin of the autocorrelation map and it is a function of $\tau$. Here, we want to exploit the possibility of following both the position and amplitude of the autocorrelation map maximum as a measure of the flow velocity and relative amount of cells taking either of the arms of a branching point. We will then extend this concept to cross correlation of different ROIs in the field of view.

We first assess the problem to reconstruct the flow along a curved vessel independent of possible ramifications. We assume that flow is occurring mainly in the SPIM plane and analyze the time stacks of images according to the STICS algorithm on an ROI (rectangular in shape) chosen in such a way to encompass a small $(40 \mu \mathrm{m})$ tract of the vessel. The particular anatomy of the zebrafish embryo justifies our assumption. We took care to orientate the embryo in such a way to image in the same light sheet $(<12 \mu \mathrm{m})$ both major vessels of the embryo. It is known (see, for example, the repository of image ${ }^{22}$ ) from the anatomy of the embryo that most of the branching occurs in the same sagittal plane.
We first evaluate, at different positions along the putative vessel axis of the STICS image at increasing lag times and fix the lag time at the maximum value, $\tau_{\text {fit }}$, for which the maximum $\vec{r}_{\max }=\left(\xi_{\max }, \eta_{\max }\right)$ of the STICS image lies within the STICS image by at least 5 to 6 pixels. The average velocity of the cells is obtained as $\vec{v}=\left(-\xi_{\max } / \tau_{\mathrm{fit}},-\eta_{\max } / \tau_{\mathrm{fit}}\right){ }^{14,23,24}$ The speed is computed, by assuming nonconstrained motion, as $d_{\max }=\sqrt{\xi_{\max }^{2}+\eta_{\max }^{2}}$.

We need to find a compromise between the finesse of the spatial reconstruction of the flow, the computation time, and the spatial resolution of the STICS image. The latter depends on the brightness of the tracers. In our hands, for red blood cells in living embryos, the minimum displacement of the STICS maximum for which the corresponding velocity uncertainty is smaller than $15 \%$ is $\left|\vec{r}_{\text {max }}\right| \cong 4$ pixels (that is $3.2 \mu \mathrm{m}$ in the sample plane). The lower the signal/noise is, the larger the minimum value of $\left|\vec{r}_{\max }\right|$ is and the coarser the reconstructed flow map is.

Once the ROI size and the fitting lag time have been chosen, the algorithm computes sequentially a set of STICS maps, updating at each step the investigation of ROI with a rigid
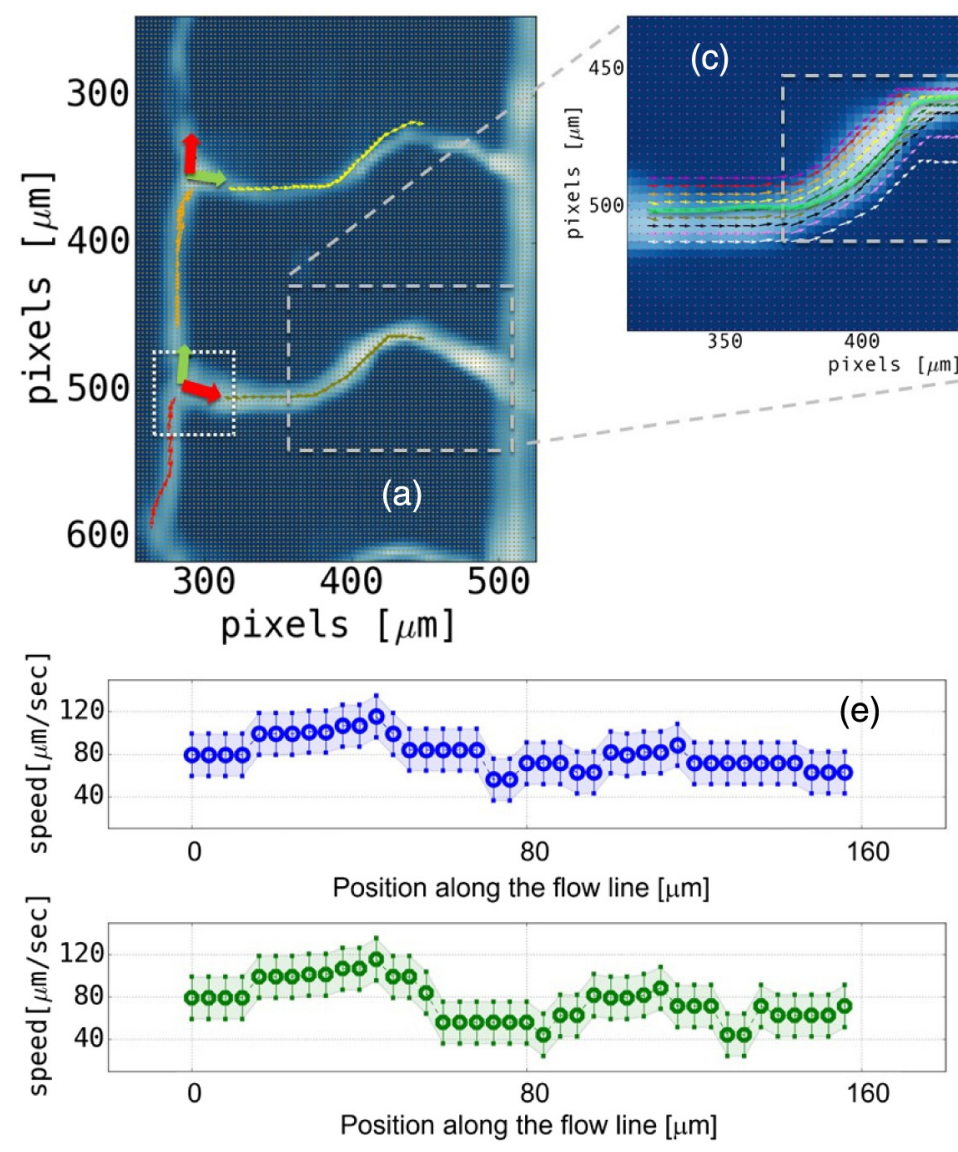
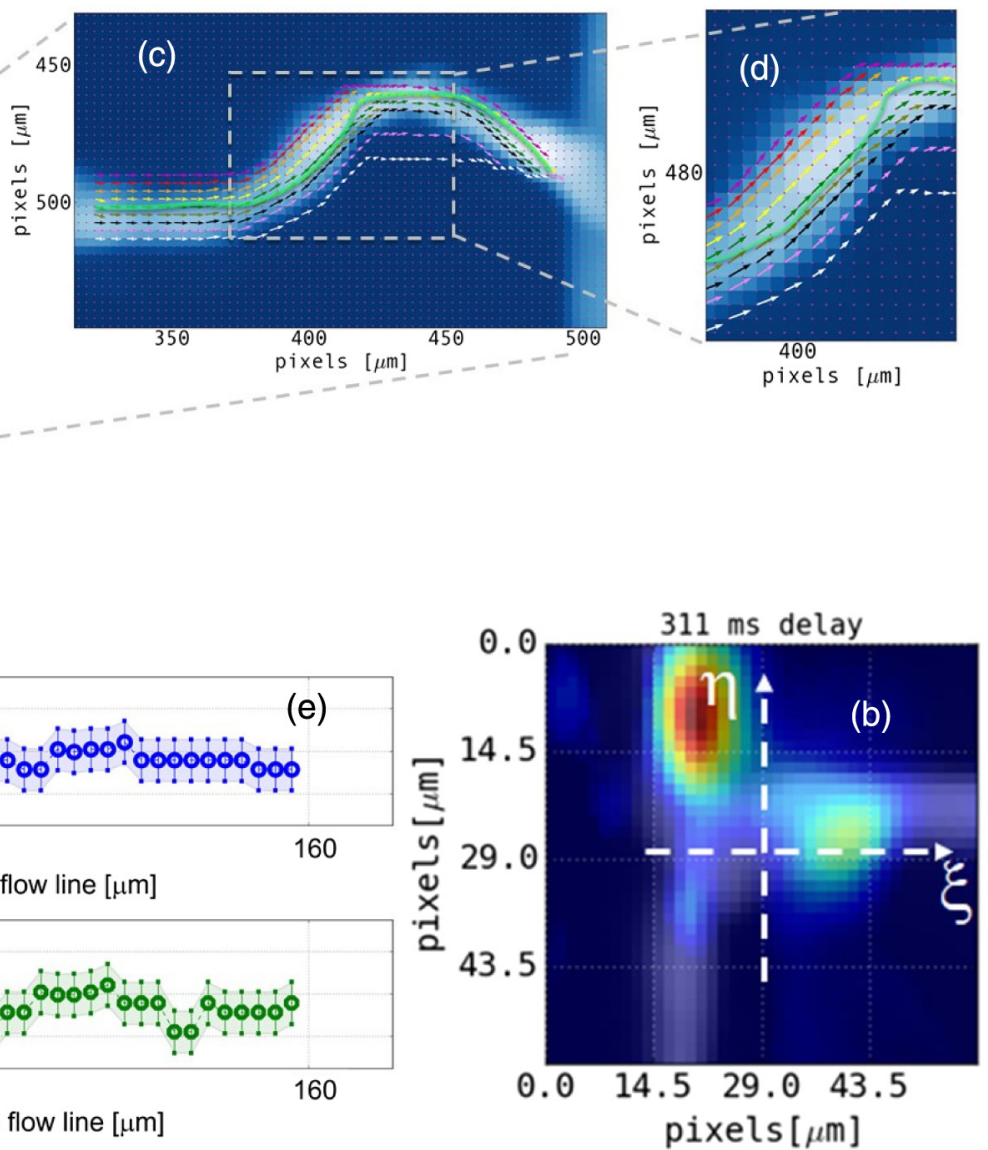

Fig. 2 Evaluation of the red blood cells flow in zebrafish embryo (3 dpf) vessels based on STICS maps from ROls of $(14 \times 14)$ pixels (pixel size $=2.9 \mu \mathrm{m})$. All the flow analyses have been done with propagation factor $\alpha=0.5$. (a) A wide field picture $\left(256 \times 256\right.$ pixels $\left.=742 \times 742 \mu \mathrm{m}^{2}\right)$ of blood circulation in the abdomen of the embryo. The flow field is reported in four linear tracts of the vessel network as thin arrows whose length is proportional to the speed. At two branching points, the dominant and marginal flow components are reported as green and red filled arrows, respectively. (b) The STICS map for the vessel branching point marked by a dotted white box in (a), at lag time $\tau=311 \mathrm{~ms}$. (c and d) Zoomed images on one intersomitic vessel in which the trajectories computed from nine different starting points have been reported together with the average flow curve (see text). (e) The flow speed along the reconstructed average flow (green) line and the adjacent (blue) line reported in (c). 
translation along the vector $\vec{T}=\alpha\left(\xi_{\max }, \eta_{\max }\right)$. The choice of small propagation factor $\alpha<1$ allows oversampling of the flow map. We typically adopt $\alpha=0.5$. A flow line can be built by assigning each flow velocity vector to the center of the corresponding ROI and by representing it with the Quiver python class. ${ }^{25}$ As can be seen from Fig. 2(a), we can follow the blood flow on a wide field of view $\left(750 \times 750 \mu \mathrm{m}^{2}\right)$ with a good resolution, $\cong 5$ to $10 \mu \mathrm{m}$ [Figs. 2(c) and 2(d)].

When approaching a branch in the vessel network, the STICS image assumes a double component character [see Fig. 2(b)]. At each step along the path (i.e., on each ROI), we evaluate the relative amplitude of the two peaks and displace the ROI along the direction that corresponds to the most relevant component (according to the maximum of each component detected in the STICS image). In this way, starting from two different points along the vessel network, we are able to evaluate the flow velocity even at the branching points [Fig. 2(a)] and follow it along the individual arms [Figs. 2(c) and 2(d)]. We observed a dependence of the resulting trajectory on the evolution parameter $\alpha$. We have found that for values $\alpha \cong 0.5$ of the evolution parameter, the flow trajectories closely follow the vessel axis, which can be measured from the morphological image [Fig. 2(a)]. There is still a dependence on the position of the starting point (the center of the first ROI of the sequence) along the putative cross section of the vessel, which is shown in Figs. 2(c) and 2(d) (different discontinuous colored lines).
However, the trajectories coalesce on a common path that we have selected by averaging the points that correspond to the position of the individual flow lines on each cross section of the vessel (at steps of $20 \mu \mathrm{m}$ ). The resulting line, reported as a thick semitransparent green line in Figs. 2(b) and 2(c), can be considered the common flow path in the selected vessel. There is no appreciable dependence of the amplitude of the flow speed on the starting point of the trajectory, which can be judged from the plot of the flow speed along two flow lines as shown in Fig. 2(e). This is to be expected since, here, we are tracking red blood cells that are approximately the size of the capillary diameter.

\subsection{Spatiotemporal Image Correlation Spectroscopy Allows Estimation of Flow Branching Ratios at the Vessel Ramifications}

The application of the STICS algorithm on a ROI that encompasses a ramification of the vessel network, such as in the black rectangle of Fig. 3(a), results in correlation images, in which two components can be singled out [Fig. 3(b)]. These components can be ascribed to the cells that take either of the two branches. The rapidity of their displacement from the center of the correlation image, $\vec{r}_{\max }^{(1)}=\left[\xi_{\max }^{(1)}, \eta_{\max }^{(1)}\right]$ and $\vec{r}_{\max }^{(2)}=\left[\xi_{\max }^{(2)}, \eta_{\max }^{(2)}\right]$, as a function of the lag times is the measure of the flow direction and the flow speed for cells flowing in each arm. The direction
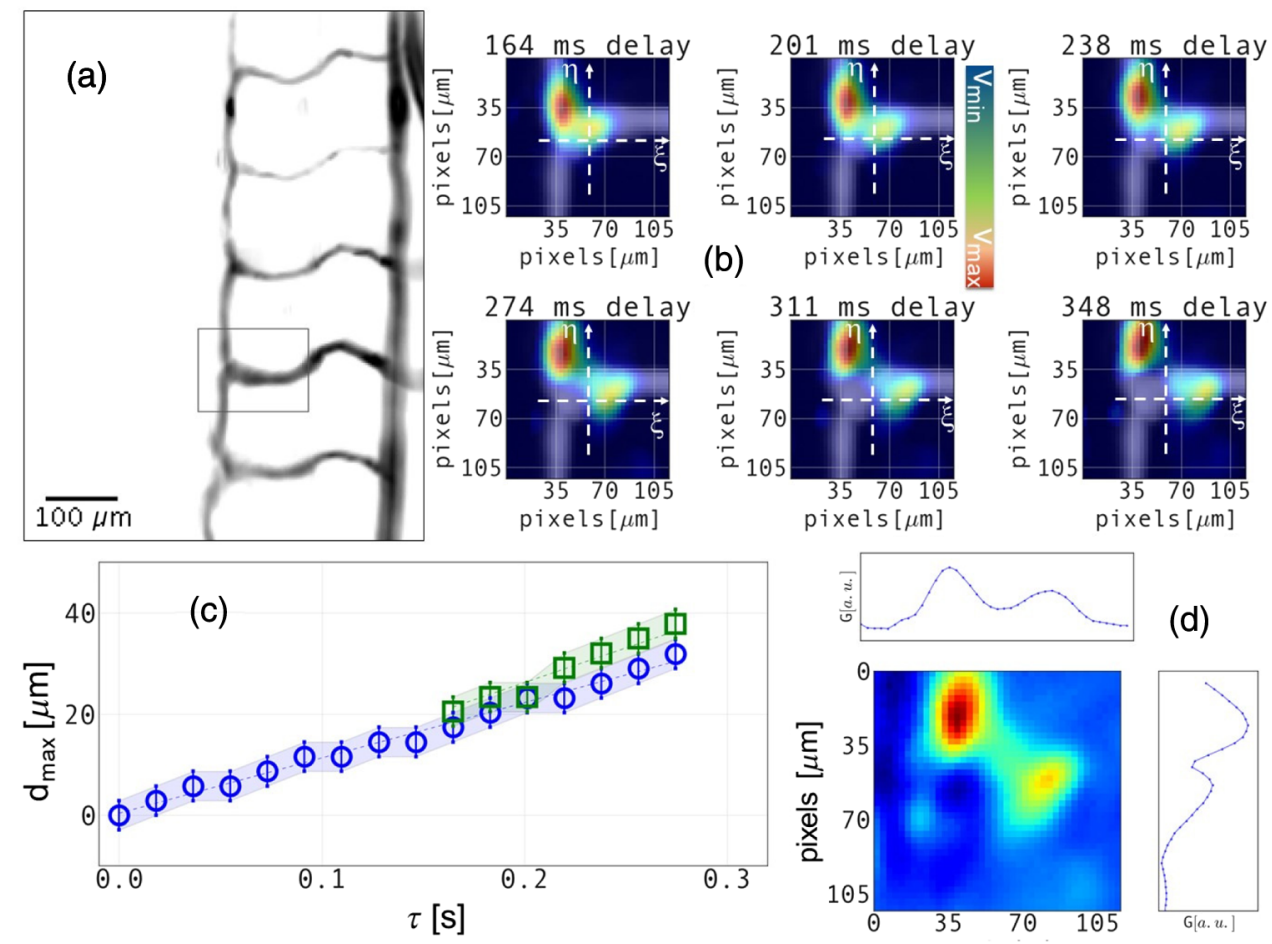

Fig. 3 Analysis of the flow ramification at branching points. (a) Morphological image taken on a zebrafish embryo (3 dpf) obtained as the standard deviation projection of the time stack of images used for the STICS analysis. (b) STICS maps computed on the ROI selected in (a) (black rectangle) for increasing lag time, from 164 to $348 \mathrm{~ms}$ from left to right and from top to bottom (flow speed coded by a "jet" LUT; morphological images superimposed in light blue). (c) Linear fit of position of the maximum of the STICS images as a function of the lag time. Open (blue) symbols refer to the prevalent component in each STICS image. Open (green) symbols refer to the marginal component (visible for lag times $\tau \geq 170 \mathrm{~ms}$ ). The linear fit to the data (dashed lines, same color code) gives velocities $v_{1}=55 \pm 1.5 \mu \mathrm{m} / \mathrm{s}$ and $v_{2}=69 \pm 3 \mu \mathrm{m} / \mathrm{s}$ for the prevalent and the marginal components, respectively. (d) The Gaussian fitting of the STICS map (lag time $=348 \mathrm{~ms}$ ) together with the profile plots along the $\xi$ and $\eta$ axes. 
of the velocity vector follows very well the morphology of the vessel ramification, which can be judged from the superposition of the correlation images with the morphological images [Fig. 3(b)]. To this purpose, the correlation images shown in Fig. 3(b) have been inverted by a central symmetry with respect to the center of the correlation space.

In the correlation maps [Fig. 3(b)], one peak was found to always be larger in magnitude irrespective of the lag time. Moreover, the standard deviation projection image (each pixel contains the standard deviation value found through the time stack) showed very similar signals on the two arms, which indicates that the emission of the individual red blood cells passing through the two arms is very similar. We assume, therefore, that the maximum amplitude of the two correlation components is proportional to the number of red blood cells flowing in one or the other ramification of the vessel branching. For example, as shown in Fig. 3(d), the brightest component of the STICS map corresponds to the red blood cells flowing upward, amounting to $66 \% \pm 1 \%$ of the overall amplitude. The marginal component represents cells flowing from left to right, amounting to $34 \% \pm 1 \%$ of the overall amplitude. The marginal component can be singled out with a reasonable signal/noise ratio $(10 \%)$ only for lag times $\tau \geq 170 \mathrm{~ms}$. Two values of the flow speed can be computed from the linear fit of the displacements of the maxima of each component as a function of the lag time, as shown in Fig. 3(c): we measure $v_{1}=55 \pm 1.5 \mu \mathrm{m} / \mathrm{s}$ and $v_{2}=69 \pm 3 \mu \mathrm{m} / \mathrm{s}$ for the prevalent and the marginal components, respectively. We find then that the marginal component is slightly faster than the prevalent one. This corresponds quite well to the observation that the leftright vessel arm is visibly larger [see Fig. 3(a)] than the bottomup arm and the fact that the vessels are approximately the size of the red blood cells diameter. It must be noticed that the algorithm for the computation of the flow velocity used here is customized to the case of right-angle crossing between vessels as in Fig. 3(a) (box). Since the flow is constrained to occur along the vessel axis, the displacement of the maximum of the autocorrelation map may, in general, not be along a line passing through the center of the map. In this case, we should compute the flow speed along the vessel axis through the incremental algorithm $\left|\vec{r}_{\max }(\tau+\delta \tau)-\vec{r}_{\max }(\tau)\right|=|\vec{v}| \delta \tau$.

\subsection{Spatiotemporal Cross-Correlation Analysis Enhance Mapping Features}

The possibility of spatially mapping the flow relies on the choice of a small ROI on which we apply the spatiotemporal correlation algorithm. The space resolution of the reconstructed flow depends on the size of the ROI (half of its linear size $=0.5 \times$ $S_{\mathrm{ROI}}$ ). However, a second key parameter for obtaining highprecision flow speed estimations is the max tracer speed $(\mathrm{K})$ inside the investigation ROI. If we assume that at least five
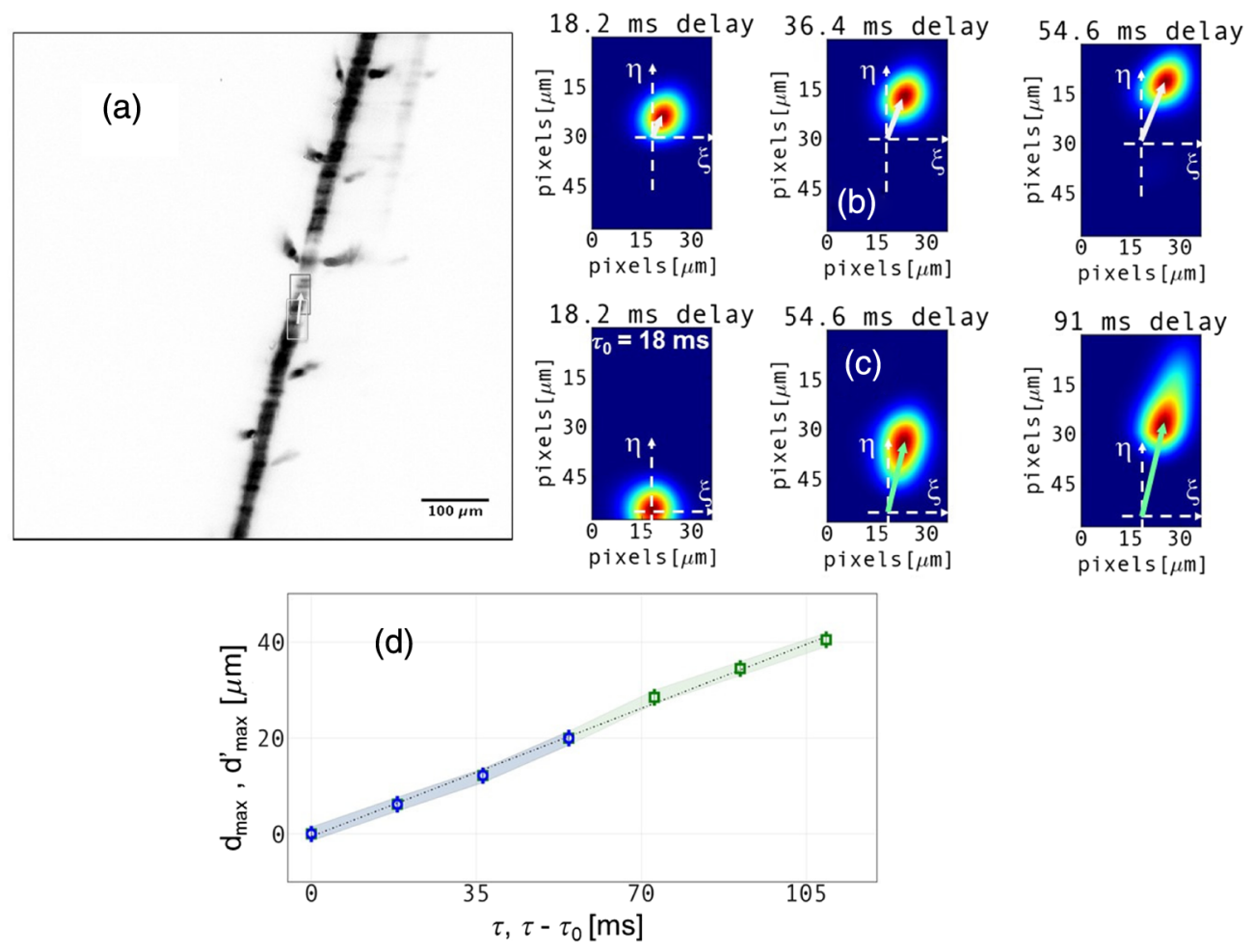

Fig. 4 Cross-correlation spatiotemporal analysis. (a) The time average of the stack of images. The light and dark gray rectangular boxes are the original and translated ROI, respectively. The white arrow indicates the translation vector. (b) Three autocorrelation images computed on the original ROI for lag times $\tau=18.2,36.4$, and $54.6 \mathrm{~ms}$. The white arrow indicates in each STICS image the displacement vector $\vec{r}_{\max }=\left(\xi_{\max }, \eta_{\max }\right)$. (c) The spatiotemporal cross-correlation maps between the original and translated ROIs for absolute lag times $\tau=18.2,54.6$, and $91 \mathrm{~ms}$. The light green arrow indicates in each crosscorrelation image the relative displacement vector $\vec{r}_{\max }^{\prime}=\left(\xi_{\max }^{\prime}, \eta_{\max }^{\prime}\right)$. (d) The plot of the displacement $d_{\max }=\sqrt{\xi_{\max }^{2}+\eta_{\max }^{2}}$ as a function of the absolute lag time $\tau$ (blue color) and $d_{\max }^{\prime}=\sqrt{\left(\xi_{\max }^{\prime}\right)^{2}+\left(\eta_{\max }^{\prime}\right)^{2}}$ as a function of the reduced lag time, $\tau-\tau_{0}$ (green color, $\tau_{0}=18 \mathrm{~ms}$ ). The dashed line is the best linear fit to the data and corresponds to the speed $v=380 \pm 9 \mu \mathrm{m} / \mathrm{s}$. 
lag time points are needed to linearly fit the relation between the displacement $\left|\vec{r}_{\max }\right|$ and the lag time, K can be estimated from the ROI size $\left(S_{\mathrm{ROI}}\right)$ and the $\mathrm{CCD}$ frame rate (fps) as $K=S_{\mathrm{ROI}} \times \mathrm{fps} / 5$. In the experiments shown in Figs. 1 and 2 , fps $=74$ frames $/ \mathrm{s}$ and $S_{\mathrm{ROI}}=16 \mu \mathrm{m}$ and we compute $K \cong 240 \mu \mathrm{m} / \mathrm{s}$. If we had larger speed values, we should increase the ROI size, losing then in spatial resolution of the flow mapping. Moreover, if the flow is not aligned with the ROI axes, we would need to increase the ROI size in both the $\xi$ and $\eta$ directions. This would increase substantially the computation time.

To solve these issues, we propose to adopt an approach consisting of two-stage analysis:

1. perform a first STICS on an ROI with a small lag time to evaluate the flow direction and

2. rigidly translate the $\mathrm{ROI}$ of half the size of the original ROI in the flow direction detected at step 1 and perform a spatiotemporal cross correlation between the original and the translated ROI [see Fig. 4(a)].

In this way, we can increase the dynamic range of the speeds that can be detected. A flow speed almost 4 times larger than in Fig. 3 can be measured on the vessel reported in Fig. 4(a) with $3 \%$ accuracy at $20-\mu \mathrm{m}$ size resolution $\left(S_{\mathrm{ROI}}=20 \mu \mathrm{m}\right)$. At the same time, we are able to keep the computational costs at the same level as the original autocorrelation algorithm. The details of the algorithm can be gained by comparing Fig. 4(b) (STICS correlation) with Fig. 4(c) (STICCS, cross correlation). We evaluate the flow velocity on the cross-correlation images by translating the correlation frame of reference $(\xi, \eta)$ at the position $\left(\xi_{0}, \eta_{0}\right)$ at which the cross-correlation peak is first detected and the lag time $\tau$ axis to the corresponding lag time, $\tau_{0}$ [see Fig. 4(c)]. The two components of the flow velocity can then be evaluated as the inverse of slope of $\xi-\xi_{0}$ and $\eta-\eta_{0}$ [the corresponding vector is reported as a white arrow in Fig. 4(c)] as a function of $\tau-\tau_{0}$. This estimate is in very close agreement with the one obtained on the autocorrelation images, which can be judged from Fig. 4(d) (open and filled symbols).

We notice that the analysis presented here is limited to 2-D flows. We could extend it to 3-D flows by following, in addition to the position of the correlation map in the correlation space, the decay with the lag time of its amplitude. The main limitation in this case could be the computation time, more than the acquisition time. In fact, we would work out the information about the out-of-plane motion from the amplitude decay of the cross correlation computed on the time stack of images of a single light sheet. This possibility is currently under investigation in our laboratory.

\section{Conclusions}

We have devised and tested correlation and cross-correlation algorithms, specifically designed for SPIM, which allow the analysis of blood flow in branched vessels. This involves the computation of the spatiotemporal correlation (STICS) and cross-correlation (STICCS) images and the analysis of the resulting maps in terms of multiple components. From the relative amplitudes of different components of the STICS map, we assign the prevalent flow direction at branching points and we are able to follow the flow at the ramification, also measuring the flow speed. The application of cross-correlation spatiotemporal methods on two different ROIs on the stacks of images allows the enhancement of the dynamic range of the flow speed measurements and mapping the flow in extended vessel networks with 5 to $10 \mu \mathrm{m}$ resolution. An example of the resulting flow map reconstruction on two consecutive branching points can be seen in Fig. 2(a). These algorithms could be a great help in analyzing a huge amount of data collected in vivo in the field of developmental biology or in vitro in the setup of vascularized microincubators for tissue engineering.

\section{Disclosures}

The authors have no relevant financial interests in this article and no potential conflicts of interest to disclose.

\section{Acknowledgments}

This work was supported by the Academic Funding Unimib 2016 (G.C. and L.S.).

\section{References}

1. D. J. Duncker and R. J. Bache, "Regulation of coronary blood flow during exercise," Physiol. Rev. 88, 1009-1086 (2008).

2. L. Hall-Stoodley, J. W. Costerton, and P. Stoodley, "Bacterial biofilms: from the natural environment to infectious diseases," Nat. Rev. Microbiol. 2(2), 95-108 (2004).

3. S. Alexander et al., "Dynamic imaging of cancer growth and invasion: a modified skin-fold chamber model," Histochem. Cell Biol. 130, 11471154 (2008).

4. P. Carmeliet, "Angiogenesis in health and disease," Nat. Med. 9, 653-660 (2003).

5. L. Sironi et al., "In vivo flow mapping in complex vessel networks by single image correlation," Sci. Rep. 4, 7341 (2014).

6. B. Zhang et al., "Biodegradable scaffold with built-in vasculature for organ-on-a-chip engineering and direct surgical anastomosis," Nat. Mater. 15(6), 669-678 (2016).

7. D. Ribatti and E. Crivellato, “'Sprouting angiogenesis," a reappraisal," Dev. Biol. 372(2), 157-165 (2012).

8. C. Korn and H. G. Augustin, "Mechanisms of vessel pruning and regression," Dev. Cell Rev. 34, 5-17 (2015).

9. P. Pozzi et al., "Electron multiplying charge-coupled device-based fluorescence cross-correlation spectroscopy for blood velocimetry on Zebrafish embryos," J. Biomed. Opt. 19(6), 067007 (2014).

10. R. M. White et al., "Transparent adult Zebrafish as a tool for in vivo transplantation analysis," Cell Stem Cell 2, 183-189 (2008).

11. L. Fieramonti et al., "Quantitative measurement of blood velocity in Zebrafish with optical vector field tomography," J. Biophotonics 8(1-2), 52-59 (2015).

12. J. Bruchmann, "Multi-channel microfluidic biosensor platform applied for online monitoring and screening of biofilm formation and activity," PLoS One 10, e0117300 (2015).

13. B. Hebert et al., "Spatiotemporal image correlation spectroscopy (STICS) theory, verification, and application to protein velocity mapping in living CHO cells," Biophys. J. 88, 3601-3614 (2005).

14. C. A. Marquezin et al., "Image cross-correlation analysis of time varying flows," Anal. Chem. 88, 7115-7122 (2016).

15. M. Bonesi et al., "Study of flow dynamics in complex vessels using Doppler optical coherence tomography," Meas. Sci. Technol. 18, 3279-3286 (2007).

16. M. Bonesi et al., "Turbulence monitoring with Doppler optical coherence tomography," Laser Phys. Lett. 4, 304-307 (2007).

17. M. Rossow, W. W. Mantulin, and E. Gratton, "Spatiotemporal image correlation spectroscopy measurements of flow demonstrated in microfluidic channels," J. Biomed. Opt. 14, 024014 (2009).

18. P. G. Pitrone et al., "OpenSPIM: an open-access light-sheet microscopy platform," Nat. Methods 10, 598-599 (2013).

19. N. D. Lawson and B. M. Weinstein, "In vivo imaging of embryonic vascular development using transgenic Zebrafish,” Dev. Biol. 248(2), 307-318 (2002). 
20. M. A. Akimenko et al., "Differential induction of four MSX homeobox genes during fin development and regeneration in Zebrafish," Development 121(2), 347-357 (1995).

21. A. Bassi et al., "In vivo label-free three-dimensional imaging of Zebrafish vasculature with optical projection tomography," J. Biomed. Opt. 16(10), 100502 (2011).

22. "Zebrafish Information Network (ZFIN)," University of Oregon, Eugene, Oregon, https://zfin.org/zf_info/anatomy.html (09 October 2017).

23. J. W. Krieger et al., "Imaging fluorescence (cross-) correlation spectroscopy in live cells and organisms," Nat. Methods 10, 1948-1974 (2015).

24. M. Bouzin et al., "An intermittent-model Fourier-space analysis of the intracellular motion of gold nanostars by scattering image correlation," Biophys. J. 109, 2246-2258 (2015).

25. J. Hunter et al., "The Matplotlib development team," https://matplotlib. org/contents.html (6 July 2017).

Nicolo G. Ceffa received his master's degree in physics from the Università degli Studi di Milano-Bicocca in 2014 and is currently a PhD student in applied physics in the physics department of this university. His research activity focuses on the use of optical microscopy and correlation techniques to investigate both in vivo and in vitro microfluidic systems, in particular, internal motions in artificial liposomes and hemodynamics studies in complex vessel networks.

Ilaria Cesana received her master's degree in physics from the Università degli Studi di Milano-Bicocca in March 2017. Her thesis was on construction and characterization of an OpenSPIM microscope used for hemodynamics investigation of model organisms, specifically zebrafish embryos (Casper).

Maddalena Collini received her PhD in physics in 1992 and is currently a professor of applied physics at the Università degli Studi di Milano-Bicocca. Her current interests involve the application of optical microscopy (super-resolution and nonlinear excitation) and image correlation techniques to the investigation of biological systems, especially metal nanoparticle uptake and transport inside cells and complex environments.

Laura D'Alfonso has been an associate professor in applied physics at the Università di Milano-Bicocca since 2014. Her research has focused on molecular biophysics, applying spectroscopic techniques to study the structural and dynamic properties of proteins, by combining experimental and theoretical-simulative approaches. She has experience in time-resolved fluorescence and advanced fluorescence techniques employing nonlinear excitation to perform high spatial and temporal resolution imaging and correlative studies, with applications in cells, tissues, and in vivo on model organisms.

Silvia Carra obtained her PhD in cellular and molecular biology from the Università degli Studi di Milano in 2007. She focused her research on developmental biology, cardiovascular development, and genes involved in human diseases using zebrafish (Danio rerio) as a model system. Currently, she works as a research collaborator for IRCCS Istituto Auxologico Italiano on neuroendocrine tumors using zebrafish as an animal model.

Franco Cotelli obtained his Laurea degree in biological sciences from the University of Milano. He is a professor of developmental biology at the University of Milano and PI of the Zebrafish Laboratory at the biosciences department. Since the 1990s, he has used the zebrafish model to study vertebrate development and genes involved in human diseases (nervous, cardiovascular, and hematopoietic systems).

Laura Sironi received her $\mathrm{PhD}$ in physics from the Università degli Studi di Milano-Bicocca in 2011 and is currently a research fellow in applied physics in the physics department of this university. She has a background in in vivo nonlinear microscopy and advanced spectroscopic methods for biological and medical applications. Her research also focuses on the development of image correlation spectroscopy techniques devoted to the study of the hemodynamics in model and complex organisms and nanoparticles-cells interaction.

Giuseppe Chirico is a professor of physics at the Department of Physics, University of Milano Bicocca, Milano, Italy. After receiving his doctorate in physics in 1990, he worked at the European Molecular Biology Laboratory (Grenoble, France), the German Cancer Research Center (DKFZ, Heidelberg, Germany) and the Laboratory for Fluorescence Dynamics (LFD, University of Illinois at UrbanaChampaign). He employs biophotonics for the study of the structure and dynamics of cells in vivo. 